\title{
Spectral Color Imaging
}

\author{
Invited
}

\author{
Jussi Parkkinen \\ Joensuu Color Group, University of Joensuu, \\ P.O.Box 111, FIN-80101 Joensuu, Finland \\ Jussi.Parkkinen@cs.joensuu.fi \\ http://cs.joensuu.fi/spectral/
}

\begin{abstract}
In fully understanding the color and its digital management, a three dimensional color representation (e.g. RGB) is not enough. The color should be represented by a n-dimensional color spectrum. Nowadays many color applications are looking for the use of color spectrum for a manageable and accurate color representation. Also new devices for spectral color acquisition and display are under investigation.

In the presentation, the spectral color approach will be introduced, spectral image analysis methods and new devices for imaging described, and application examples shown. The term spectral instead of multispectral is used. This follows the logic where the binary image has binary value in each pixel and gray-level image has a gray-value in each pixel. Since in the images we are interested have a spectrum in each pixel, we call them spectral images. In e.g. remote sensing these imagesare called multi- or hyperspectral images.
\end{abstract}

\section{Introduction to Spectral Color Research}

Color science has mainly concentrated on the research problems, which are based on the trichromaticity model of human visual system. In this model, the idea is that the color vision is based on three color sensitive sensors (in human vision cone cells), which have different wavelength sensitivity with each other. In the physical sense, light is a part of the electromagnetic spectrum. According to the trichromaticity model, the wavelength range roughly from $400 \mathrm{~nm}$ to $700 \mathrm{~nm}$ defines the color of an object. Vast majority of standard color representations and existing devices are based on this model.

Another, a general approach to the color science emerged in 80's. According to this idea, the color is analyzed based on the color spectrum reaching the color detection system. This signal is called color signal. Using this approach, we are not restricted into the human visual range. This is important in understanding other biological color vision systems. For example solitary bee has ultraviolet sensitive cells in it's eye. This general approach helps also in designing artificial visual systems, for example in an industrial quality control problems accurate 
color information may be required on yellow colors but no color information is needed elsewhere. The general approach gives possibility to design an adaptive color analysis system with maximum accuracy in yellow colors.

It was shown first time in mid 80's that three components are not enough to represent color information. Now this is a commonly accepted finding and the number of components to represent color rather accurately is 5-8. The above mentioned general approach to the color science is a common basis for the color research. New devices and tools, however, are required in order to get all the benefits out of this general approach.

Using the general approach as well as devices and tools based on it, the color science will provide a deeper understanding to the nature of color, also in digital domain, and forward new applications ranging from paper industry to eCommerce.

Some of the key areas of present research in spectral color science can be found in the following list of Joensuu Color Group research interests.

\section{- Accurate color measurement and physical basis of color}

The goal is to study methods for accurate color measurements. The measurements are made on a point in an object. It has been shown that the external measurement conditions should be well defined for an accuracy comparable to an experienced observer to be reached. The measurements are expanded into the UV-region and measurement of fluorescent. Also, thermochromism is a topic of interest, including thermochromism of fluorescent objects.

\section{- The structure and coordinate systems in n-dimensional color space}

This is the first subtopic, we started color research with. The main findings in this topic are that we showed that the dimensionality of color space is more than three. From that, we have studied different methods for color representation and color coordinate systems. A much used tool in this research is the Principal Component Analysis (PCA).

\section{- Spectral image acquisition}

In the spectral image acquisition, there is a special problem: the object to be imaged is actually three dimensional (two spatial and a spectral dimension), but the imaging sensors are two dimensional (normally CCD-arrays). This means that in the imaging device one has to scan in either the spectral or one spatial dimension. We have studied methods and constructed prototypes of spectral imager based on spectral scanning using optimal spectral filter. This means e.g. finding optimal subspace of the color space so, that the subspace can be spanned by positive basis vectors. This is a benefit in optical realization of the filters.

\section{- Connections to the biological visual systems}

We have studied the artificial visual systems by using "biological hardware". We have build a protein based artificial retina. The protein belongs to the family of rhodopsins, the proteins which are responsible in light detection in 
animal eye. The protein is modified by biotechnological methods so that we have more than one different spectral sensitivities. The detector element is made by immobilizing the proteins into a polymer matrix. The protein gives an electrical signal for a light impulse. These response signals of modified proteins are used in color detection.

\section{- Compression of digital spectral images}

The digitized spectral images requires much memory if they are represented as raw images. By using the general knowledge about the structure and representation methods of color space, we have developed methods for spectral image compression. We have also introduced a new concept of spectral video, where each video frame is a spectral image. It requires also efficient methods to compress spectral video for data communication purposes.

\section{- Applications of spectral imaging}

Earlier, all the applications of color research were based on measurements of color in a single point or region. Since the spectral imaging devices have become available, also the applications of spectral images have emerged. These include topics like industrial color quality control, use of spectral images in conservation of cultural heritage, 3D-spectral imaging, medical applications, and e-commerce.

\section{References}

1. Wyszecki, G. and Stiles, W. S., Color science : concepts and methods, quantitative data and formulae, John Wiley \& Sons., New York, USA, 2000

The basic of color science. It covers all aspects of traditional color science, including a number of standards tabulated. The book does not cover color images but the color on homogenous regions.

2. Hardeberg J., Acquisition and Reproduction of Color Images: Colorimetric and Multispectral approaches, Dissertation.com, Parkland, USA, 2001.

This Hardeberg's Ph.D. thesis is first published book about the multispectral color imaging. It gives a good overview about the multispectral approach to the color.

3. Berns R., Billmeyer and Salzman's Principles of Color Technology (third ed.), John Wiley \& Sons, Bew York, USA, 2000.

Illustrative and easy to read book and express clearly the principles color and it's management.

4. Gegenfurtner K. and Sharpe L. (Eds.), Color vision : from genes to perception, Cambridge University Press, Cambridge, U.K., 1999.

The book gives good overview of biological visual system from color vision point of view. Also the spectral approach is taken into account. 
5. Pratt, William K., Digital Image Processing, John Wiley \& Sons, New York, USA, 2001.

A book, which has probably the widest chapter about color among the digital image processing books. Normally, in the digital image processing books color images are explained rather shortly, and in traditional $3 D$ color spaces.

6. Fairchild, Mark, Color appearance models, Addison-Wesley, Reading, USA, 1998.

Explains clearly the important topics taken into account in color image output.

Following are some books about color. Normally they cover in the physical principles part, the visible region spectrum, but the color is expressed in a traditional way.

7. Green P. and MacDonald L. (Eds.), Colour engineering : achieving device independent colour, Wiley, Chichester, U.K., 2002.

8. Hunt R.W.G., Measuring Colour, Ellis Horwood, Chichester, U.K., 2001.

9. Kang H., Digital Color Halftoning, SPIE Optical Engineering Press, Bellingham, USA, 1999.

10. MacDonald L. and Luo R. (Eds.), Colour image science : exploiting digital media Wiley, Chichester, U.K., 2002.

11. Malacara, Daniel, Color vision and colorimetry : theory and applications, SPIE Press, Bellingham, USA, 2002.

12. Nassau Kurt (ed.), Color for science, art and technology, Elsevier, Amsterdam, The Netherlands, 1998.

13. Plataniotis K., Color image processing and applications, Springer Verlag, Berlin, Germany, 2000.

14. Sharma G. (Ed.), Digital Color Imaging Handbook, CRC Press, Boca Raton, USA, 2002.

15. Wandell Brian, Foundations of vision, Sinauer Associates, Sunderland, USA, 1995.

16. Zollinger H., Color a multidisciplinary approach, Verlag Helvetica Chimica Act, Zuerich, Switzerland, 1999.

Some scientific journals, where spectral color papers are published

17. Color Research and Applications

18. Journal of Optical Society of America A

19. IEEE tr on Image Processing

20. Journal of Electronic Imaging

\section{Conferences}

21. Multispectral Color Science, MCS: annually in different countries

22. European Conference on Color in Graphics, Imaging, and Vision, CGIV: biannually in Spring time in Europe, 2004 in Aachen

23. Color Imaging Conference, Scottsdale, AZ, USA: annually in November in Scottsdale

Many conferences include a session in spectral image processing.

Two Web-pages

24. http://www.multispectral.org : an unofficial web page of the (multi)spectral color science

25. http://cs.joensuu.fi/spectral : the University of Joensuu Color Group home page, spectrum databases, some theses, Matlab and C-programs, demos about color spectrum, links 